\title{
INTERVENÇÕES PARA O ESTRESSE E ANSIEDADE NA ENFERMAGEM: REVISÃO INTEGRATIVA
}

\section{INTERVENTIONS FOR STRESS AND ANXIETY IN NURSING: INTEGRATIVE REVIEW}

\author{
Andréia Barcellos Teixeira Macedo ${ }^{1} *$ Edwing Alberto Urrea Veja $^{2} *$ Liliana Antoniolli $^{3}$ \\ Jessica Morgana Gediel Pinheiro ${ }^{4} *$ Thayane Martins Dornelles $^{5} *$ Sônia Beatriz Cocaro de \\ Souza $^{6}$
}

\begin{abstract}
RESUMO
Objetivo: Identificar intervenções utilizadas para redução do estresse e ansiedade em profissionais da enfermagem e sintetizar respectivos resultados. Método: Revisão integrativa realizada nas bases MEDLINE/Pubmed, SCOPUS, WoS, LILACS, SCIELO, EMBASE e EBSCO CINAHL. Foram incluídas publicações de janeiro de 2016 a dezembro de 2020, em inglês, espanhol e português. Resultados: Selecionou-se 27 publicações, com intervenções diversificadas, realizadas como terapia única ou em conjunto com outras atividades. Houve redução dos desfechos em 15 (55,5\%) das publicações. Conclusão: Constatou-se que algumas intervenções podem auxiliar na redução do estresse e ansiedade nos profissionais de enfermagem. Entretanto, identificou-se a necessidade de mais estudos frente à dimensão do problema.
\end{abstract}

Palavras-chave: Ansiedade; Estresse Ocupacional; Enfermagem; Terapias Complementares; Revisão.

\begin{abstract}
Objective: To identify interventions used to reduce stress and anxiety in nursing professionals and synthesize their results. Method: Integrative review performed in MEDLINE/Pubmed, SCOPUS, WoS, LILACS, SCIELO, EMBASE and EBSCO CINAHL databases. Publications from January 2016 to December 2020, in English, Spanish and Portuguese were included. Results: We selected 27 publications, with diversified interventions, performed as a single therapy or in conjunction with other activities. There was a reduction in outcomes in $15(55.5 \%)$ of the publications. Conclusion: It was found that some interventions can help reduce stress and anxiety in nursing professionals. However, the need for further studies was identified regarding the dimension of the problem.

Keywords: Anxiety; Occupational Stress; Nursing; Complementary Therapies; Review.
\end{abstract}

\footnotetext{
${ }^{1}$ Enfermeira, mestre em enfermagem, doutoranda do Programa de Pós-graduação em Enfermagem da Universidade Federal do Rio Grande do Sul. ORCID: https://orcid.org/0000-0003-4219-4731

${ }^{2}$ Enfermeiro, mestre em enfermagem, doutorando do Programa de Pós-graduação em Enfermagem da Universidade Federal do Rio Grande do Sul. ORCID: https://orcid.org/0000-0002-9578-4252

${ }^{3}$ Enfermeira, mestre em enfermagem, doutoranda do Programa de Pós-graduação em Enfermagem da Universidade Federal do Rio Grande do Sul. ORCID: https://orcid.org/0000-0003-0806-9910

${ }^{4}$ Enfermeira, mestranda do Programa de Pós-graduação em Enfermagem da Universidade Federal do Rio Grande do Sul. ORCID: https://orcid.org/0000-0003-1530-7198

${ }^{5}$ Enfermeira, mestre em enfermagem. ORCID: https://orcid.org/0000-0001-7377-7054

${ }^{6}$ Enfermeira, doutora em ciências médicas: psiquiatria, Faculdade de Medicina da UFRGS, docente do Programa de Pós-graduação em Enfermagem da Universidade Federal do Rio Grande do Sul. ORCID: https://orcid.org/0000-0001-9394-5465
} 


\section{INTRODUÇÃO}

A equipe de enfermagem se encontra constantemente exposta a estressores laborais e a preocupação com a saúde mental destes profissionais tem sido tema em diversos fóruns de discussão. $\mathrm{O}$ contato diário com situações desgastantes, sem mecanismos de proteção e enfrentamento adequados por parte dos trabalhadores, pode desencadear uma série de sintomas físicos e psicológicos, e enfermidades graves e crônicas, como problemas psicossomáticos, neurológicos e cardiovasculares, além de esgotamento profissional $^{(1)}$.

O cuidado contínuo de pacientes graves e o convívio com a dor, o sofrimento e a morte no contexto laboral, expõe os profissionais a situações estressoras que contribuem para ocorrência dos sintomas de ansiedade aguda ou crônica. O estresse desgasta o indivíduo devido à dificuldade em mobilizar recursos de acordo com a demanda exigida no trabalho, e a ansiedade esgota pela antecipação das necessidades que ainda não ocorreram $^{(1)}$.

\section{Indivíduos constitucionalmente} ansiosos podem possuir a tendência de reagir de maneira excessiva ao estresse, à probabilidade de dano e a subestimar a própria capacidade para lidar com as situações potencialmente ameaçadoras ${ }^{(2)}$. A ansiedade passa a ser tratada como enfermidade quando é exagerada e desproporcional ao estímulo, ou até mesmo quando distorce

substancialmente do estado real do indivíduo, podendo prejudicar o raciocínio, o desempenho de atividades diárias e do trabalho ${ }^{(3)}$.

Sabe-se que o estresse ocupacional e ansiedade acometem os profissionais de enfermagem a nível mundial. No ranking de países mais estressados, o Brasil figura na segunda posição e só perde para o Japão. Estudo realizado em 2017 verificou que 70\% dos brasileiros participantes apontaram o trabalho como fator que mais causa estresse ${ }^{(4)}$. Por sua vez, os distúrbios de ansiedade afetam 9,3\% das pessoas que vivem no Brasil. Estudo realizado com trabalhadores da enfermagem identificou que $44,4 \%$ apresentavam algum nível de ansiedade, principalmente técnicos de enfermagem $^{(5)}$.

Desta forma, intervenções a nível individual, coletivo e organizacional são necessárias para minimizar os efeitos do estresse crônico e da ansiedade. Fortalecer o individual para reduzir os riscos do coletivo e da organização. Na enfermagem, estratégias que identifiquem e reduzam sintomas de estresse e ansiedade gerados no ambiente de trabalho devem fazer parte do gerenciamento de recursos humanos através dos órgãos de saúde ocupacional $^{(1,6)}$.

Frente ao exposto, a justificativa para esta investigação se baseia na importância de 
sintetizar e divulgar o conhecimento sobre intervenções que já tenham sido testadas na redução do estresse e da ansiedade nos profissionais da enfermagem, fornecendo subsídios para organização de atividades de prevenção e promoção da saúde destes trabalhadores. O estudo objetivou identificar intervenções utilizadas para redução do estresse e ansiedade em profissionais da enfermagem e sintetizar respectivos resultados.

\section{MÉTODO}

Trata-se de uma revisão integrativa (RI) da literatura, estruturada de acordo com as seguintes etapas: estabelecimento da questão norteadora, amostragem, extração de dados, avaliação crítica, análise e síntese dos estudos, e síntese do conhecimento ${ }^{(7)}$.

A questão norteadora foi construída com apoio da estratégia $\mathrm{PICO}^{(8)}$, onde a população $(\mathrm{P})$ considerada foi profissionais da enfermagem, a intervenção (I) quaisquer intervenções testadas, comparação (C) não foi aplicado, e resultados/desfechos (O) o efeito da intervenção sobre estresse e ansiedade. Desta forma, definiu-se como questão norteadora: "Quais intervenções foram utilizadas para redução do estresse e ansiedade em profissionais da enfermagem e quais resultados obtidos com estas intervenções?"

Os descritores e seus respectivos sinônimos foram selecionados através de pesquisa no Medical Subject Headings (MESH) e Descritores em Ciências da Saúde (DECS), e combinados através dos operadores boleanos $O R$ e $A N D$, conforme apresentado no Quadro 1. Os termos foram pesquisados no título e resumo das publicações.

Quadro 1. Estratégias de busca nas bases de dados. Porto Alegre, RS, Brasil, 2021.

\begin{tabular}{|c|c|}
\hline WoS & $\begin{array}{l}\text { TÓPICO: ((Nurses) OR (Registered Nurse) OR (Nursing Personnel) OR } \\
\text { (Nurs*)) AND TÓPICO: ((Occupational Stress) OR (Job Stress) OR (Stress, } \\
\text { Workplace)) AND TÓPICO: ((Anxiety) OR (Social Anxiety)) Tempo } \\
\text { estipulado: 2016-2020. Índices: SCI-EXPANDED, SSCI, A\&HCI, CPCI-S, } \\
\text { CPCI-SSH, ESCI. }\end{array}$ \\
\hline EMBASE & $\begin{array}{l}\text { ('nurse' OR 'registered nurse' OR 'nursing personnel' OR 'nurs*') AND } \\
\text { ('occupational stress' OR 'job stress' OR 'stress, workplace') AND ('anxiety' OR } \\
\text { 'social anxiety') AND [2016-2020]/py }\end{array}$ \\
\hline
\end{tabular}




\begin{tabular}{|c|c|}
\hline CINAHL & $\begin{array}{l}\text { ("Nurses" OR "Registered Nurse" OR "Nursing Personnel" OR “Nurs*”) } \\
\text { AND ("Occupational Stress" OR "Job Stress" OR "Stress, Workplace") } \\
\text { AND ( "Anxiety" OR "Social Anxiety") }\end{array}$ \\
\hline SCOPUS & $\begin{array}{l}\text { (ALL (\{Nurses }\} \text { OR }\{\text { Registered Nurse }\} \text { OR }\{\text { Nursing Personnel }\} \text { OR } \\
\{\text { Nurs* }\}) \text { AND ALL (\{Occupational Stress }\} \text { OR }\{\text { Job Stress }\} \text { OR } \\
\{\text { Stress, Workplace }\}) \text { AND ALL (\{Anxiety }\} \text { OR \{Social Anxiety }\})) \\
\text { AND (LIMIT-TO (PUBYEAR, 2020) OR LIMIT-TO ( PUBYEAR , } \\
\text { 2019) OR LIMIT-TO (PUBYEAR, 2018) OR LIMIT-TO (PUBYEAR, } \\
\text { 2017) OR LIMIT-TO (PUBYEAR, 2016)) }\end{array}$ \\
\hline LILACS & $\begin{array}{l}\text { tw:(("Stress, Psychological" OR “occupational stress”) AND nurs* AND } \\
\text { "anxiety”) AND (instance:"regional") AND (mj:("Estresse Psicológico" } \\
\text { OR "Adaptação Psicológica" OR "Recursos Humanos de Enfermagem } \\
\text { no Hospital" OR "Enfermeiras e Enfermeiros" OR "Atitude do Pessoal } \\
\text { de Saúde" OR "Qualidade de Vida") AND la:("en" OR "pt" OR "es") } \\
\text { AND year_cluster:("2016" OR "2017" OR "2018" OR "2019" OR } \\
\text { "2020")) }\end{array}$ \\
\hline EBSCO CINAHL & 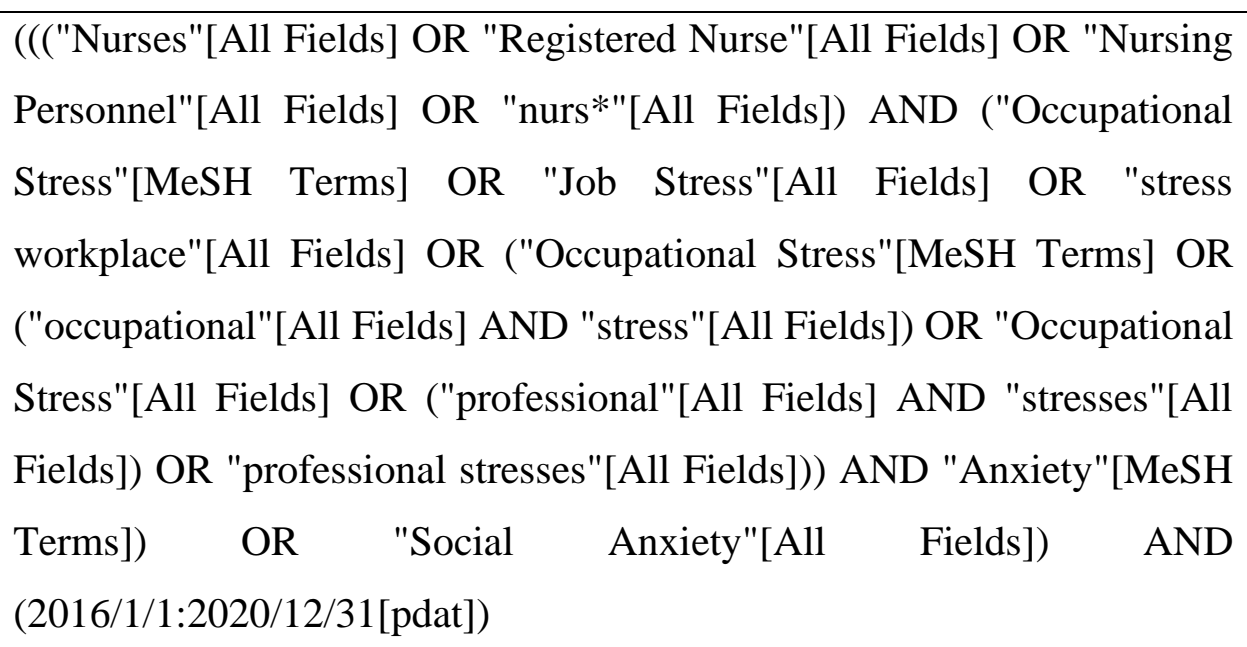 \\
\hline SCIELO & $\begin{array}{l}\text { TÓPICO: ((Nurses) OR (Registered Nurse) OR (Nursing Personnel) } \\
\text { OR (Nurs*)) AND TÓPICO: ((Occupational Stress) OR (Job Stress) } \\
\text { OR (Stress, Workplace)) AND TÓPICO: ((Anxiety) OR (Social } \\
\text { Anxiety)) Tempo estipulado: 2016-2020. Índices: SCIELO. }\end{array}$ \\
\hline
\end{tabular}

Fonte: os autores, 2021. 
Foram incluídos artigos primários, publicados de janeiro de 2016 a dezembro de 2020, nos idiomas português, inglês e espanhol e que respondessem à questão norteadora, e excluídas as publicações apresentadas em formato de tese, dissertação, manuais, capítulos de livros, reflexões, revisões, editais, opiniões e comentários de especialistas, bem como artigos duplicados nas bases de dados.

A coleta de dados foi feita nas bases Medical Literature Analysis and Retrieval System Online (MEDLINE via PubMed), Web of Science (WoS), Excerpta Medica Database (EMBASE), Cumulative Index to Nursing and Allied Health Literature (CINAHL), SCOPUS (Elsevier), Literatura Latino-Americana e do Caribe em Ciências da Saúde (LILACS) via Biblioteca Virtual em Saúde (BVS), EBSCO CINAHL, e na biblioteca Scientific Electronic Library Online (SCIELO). A escolha das bases de dados considerou a abrangência e afinidade com o tema. As produções foram acessadas por meio do portal de periódicos da Coordenação de Aperfeiçoamento de Pessoal de Nível Superior (CAPES).

A seleção dos estudos foi realizada por dois revisores independentes, com posterior checagem das inconsistências e consenso entre as partes. Quando em situação de divergência, um terceiro revisor foi consultado. As publicações foram exportadas para o software Excel® e organizadas e sumarizadas através de formulário construído pelos autores.

Os dados estão apresentados por meio de análise descritiva. Para a síntese do efeito das intervenções testadas, considerou-se o resultado estatístico apresentado nas publicações. Respeitaram-se os aspectos éticos, com citação fidedigna das fontes e definições dos autores.

\section{RESULTADOS}

A amostra foi composta por 27 publicações, conforme apresentado na Figura 1, construída com apoio do protocolo Preferred Reporting Items for Systematic reviews and Meta-Analyses (PRISMA) ${ }^{(9)}$. Foram $21(77,8 \%)$ ensaios clínicos e seis $(22,2 \%)$ estudos do tipo quase experimental, $12(44,4 \%)$ eram da WoS, nove $(33,4 \%)$ do SCOPUS, três $(11,1 \%)$ da MEDLINE/PubMed, dois $(7,4 \%)$ da EMBASE e um $(3,7 \%)$ do EBSCO CINAHL. 
Figura 1 - Fluxograma de seleção dos estudos, elaborado a partir da recomendação PRISMA. Porto Alegre, RS, Brasil, 2021.

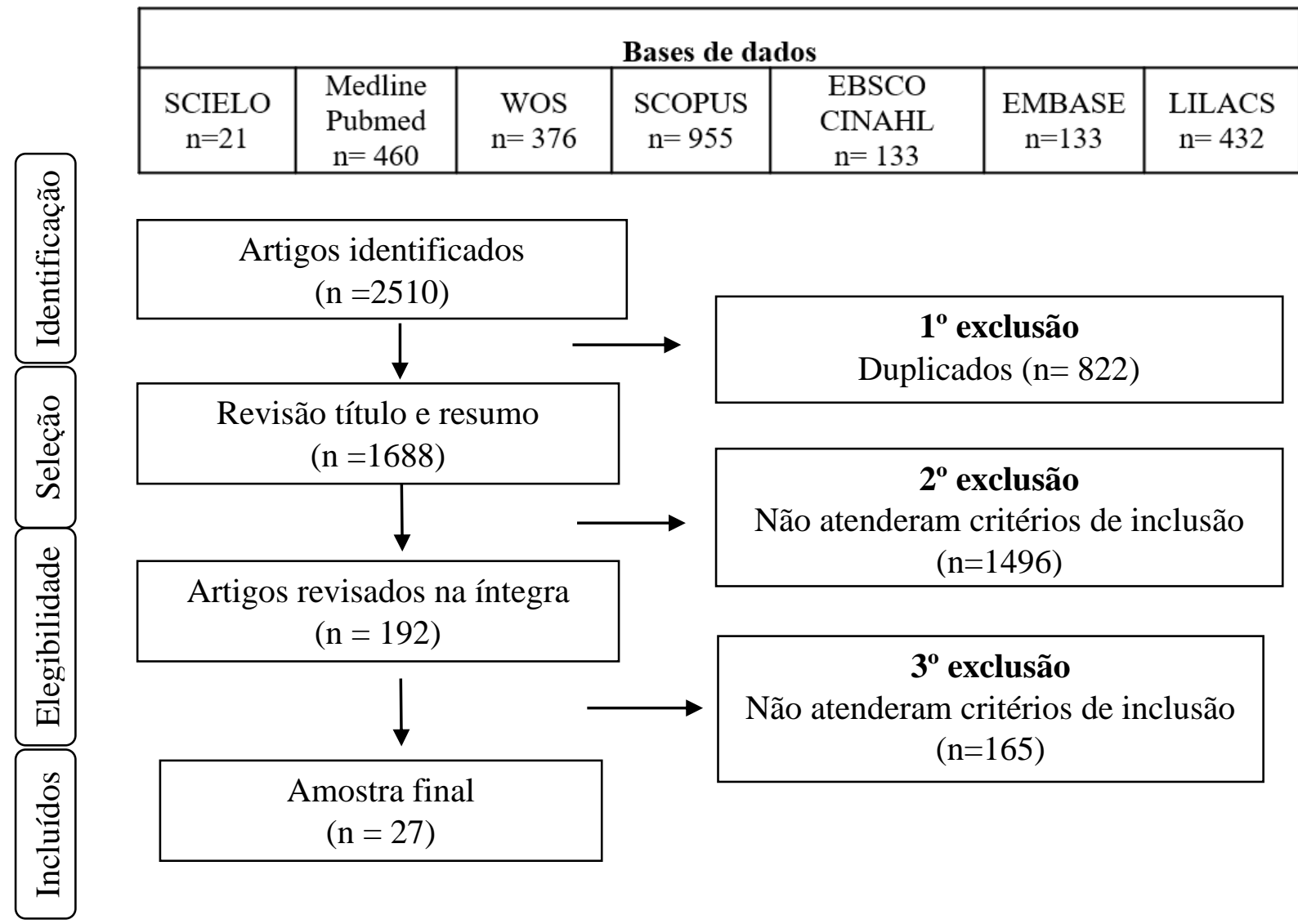

Fonte: Os autores.

O Quadro 2 apresenta a síntese das publicações, separadas didaticamente conforme o desfecho de interesse: 1) Estresse,
2) Ansiedade e 3) Estresse e Ansiedade, com respectivamente dez $(37 \%)$, cinco $(18,5 \%)$ e $12(44,5 \%)$ estudos.

Quadro 2 - Caracterização dos estudos primários segundo país, ano de publicação, tipo de estudo, objetivo e população estudada. Porto Alegre, RS, Brasil, 2021.

\begin{tabular}{|c|c|c|}
\hline \multicolumn{3}{|c|}{ 1) Desfecho: Estresse } \\
\hline Cod. & País e ano & Objetivo e tipo estudo \\
\hline $\mathrm{A} 1^{(10)}$ & $\begin{array}{l}\text { USA } \\
2016\end{array}$ & $\begin{array}{l}\text { Avaliar a eficácia do programa Stress Management for Nurses } \\
(B R E A T H E) \text { em enfermeiros de seis hospitais; ECR }\end{array}$ \\
\hline
\end{tabular}




\begin{tabular}{|c|c|c|}
\hline $\mathrm{A} 2^{(11)}$ & $\begin{array}{l}\text { Brasil } \\
2018\end{array}$ & $\begin{array}{l}\text { Verificar a efetividade da aromaterapia associada à massagem na equipe } \\
\text { de enfermagem do centro cirúrgico; ECR }\end{array}$ \\
\hline $\mathrm{A} 3^{(12)}$ & $\begin{array}{l}\text { India } \\
2019\end{array}$ & $\begin{array}{l}\text { Descobrir o efeito do Mahamantra Chanting no estresse em enfermeiros } \\
\text { de um hospital; ECR }\end{array}$ \\
\hline $\mathrm{A} 4^{(13)}$ & Irã & $\begin{array}{l}\text { Avaliar a eficácia da Terapia Comportamental (TCC) no estresse de } \\
\text { enfermeiros intensivistas; ECR }\end{array}$ \\
\hline $\mathrm{A} 5^{(14)}$ & $\begin{array}{l}\text { Brasil } \\
2020\end{array}$ & $\begin{array}{l}\text { Avaliar o efeito do relaxamento muscular no estresse e bem-estar no } \\
\text { trabalho em enfermeiros da emergência e hemodinâmica; QE }\end{array}$ \\
\hline $\mathrm{A} 6^{(15)}$ & $\begin{array}{l}\text { Coréia do Sul } \\
\qquad 2020\end{array}$ & $\begin{array}{l}\text { Investigar os efeitos da inalação de patchouli no estresse, qualidade de } \\
\text { vida profissional, pressão arterial e frequência cardíaca em enfermeiros } \\
\text { emergencistas; ECR }\end{array}$ \\
\hline $\mathrm{A} 7^{(16)}$ & $\begin{array}{l}\text { USA } \\
2020\end{array}$ & $\begin{array}{l}\text { Explorar o impacto do Jin Shin Jyutsu Self-Help (JSJ) no estresse e na } \\
\text { eficácia do cuidado de enfermagem de um hospital; ECR }\end{array}$ \\
\hline $\mathrm{A} 8^{(17)}$ & $\begin{array}{l}\text { India } \\
2020\end{array}$ & $\begin{array}{l}\text { Examinar o impacto de cantar o Mahamantra na redução do estresse entre } \\
\text { enfermeiras de um hospital; ECR }\end{array}$ \\
\hline A9 ${ }^{(18)}$ & Irã & $\begin{array}{l}\text { Determinar os efeitos do treinamento em gerenciamento do estresse de } \\
\text { enfermeiros hospitalares; ECR }\end{array}$ \\
\hline $\mathrm{A} 10^{(19)}$ & $\begin{array}{l}\text { Irã } \\
2020\end{array}$ & $\begin{array}{l}\text { Avaliar a eficácia de um workshop sobre gerenciamento do tempo no } \\
\text { estresse para enfermeiros emergencistas; ECR }\end{array}$ \\
\hline
\end{tabular}

\section{2) Desfecho: Ansiedade}

\begin{tabular}{|c|c|l|}
\hline A11 & Brasil & $\begin{array}{l}\text { Avaliar o efeito do Protocolo Auricular para Dor e Ansiedade (APPA) na } \\
\text { enfermagem de um hospital; ECR }\end{array}$ \\
\hline A12(21) & Grécia & $\begin{array}{l}\text { Examinar os efeitos de musicoterapia na ansiedade e depressão em } \\
\text { enfermeiros oncologistas; ECR }\end{array}$ \\
\hline
\end{tabular}




\begin{tabular}{|l|c|l|}
\hline A13 & $\begin{array}{l}\text { Japão } \\
2019\end{array}$ & $\begin{array}{l}\text { Analisar a eficácia de treinamento com Mindfulness na saúde mental dos } \\
\text { enfermeiros hospitalares; ECR }\end{array}$ \\
\hline A14 & Irã & $\begin{array}{l}\text { Analisar a eficácia da logoterapia na ansiedade e no burnout de } \\
\text { enfermeiros hospitalares; QE }\end{array}$ \\
\hline A15 & 2019 & $\begin{array}{l}\text { Avaliar se colorir mandalas é eficaz na redução da ansiedade da } \\
\text { enfermagem médico-cirúrgica; QE }\end{array}$ \\
\hline
\end{tabular}

\section{3) Desfecho: Estresse e Ansiedade}

\begin{tabular}{|c|c|c|}
\hline $\mathrm{A} 16^{(25)}$ & $\begin{array}{l}\text { Brasil } \\
2016\end{array}$ & $\begin{array}{l}\text { Avaliar os efeitos do Stress Reduction Program (SRP) na enfermagem de } \\
\text { um hospital escola; QE }\end{array}$ \\
\hline $\mathrm{A} 17^{(26)}$ & $\begin{array}{l}\text { Turquia } \\
2017\end{array}$ & $\begin{array}{l}\text { Investigar os efeitos da aromaterapia no estresse e ansiedade em } \\
\text { enfermeiros intensivistas; ECR }\end{array}$ \\
\hline $\mathrm{A} 18^{(27)}$ & $\begin{array}{l}\text { USA } \\
2017\end{array}$ & $\begin{array}{l}\text { Avaliar a eficácia do programa Stress Management and Resilience } \\
\text { Training Program (SMART) no estresse e burnout de enfermeiros em um } \\
\text { centro médico; QE }\end{array}$ \\
\hline A19 $(28)$ & $\begin{array}{l}\text { USA } \\
2017\end{array}$ & $\begin{array}{l}\text { Medir os efeitos do Relaxation Response na ansiedade, depressão, bem- } \\
\text { estar e estresse de enfermeiros cardiologistas; ECR }\end{array}$ \\
\hline $\mathrm{A} 20^{(29)}$ & $\begin{array}{l}\text { Austrália } \\
2018\end{array}$ & $\begin{array}{l}\text { Avaliar a eficácia do Brief Mindful Self-Care and Resiliency (MSCR) na } \\
\text { fadiga por compaixão e ansiedade de enfermeiros de um hospital escola; } \\
\text { ECR }\end{array}$ \\
\hline $\mathrm{A} 21^{(30)}$ & $\begin{array}{l}\text { China } \\
2018\end{array}$ & $\begin{array}{l}\text { Investigar o efeito do Mindfulness-Based Stress Reduction(MBSR) no } \\
\text { estresse e saúde mental de enfermeiros psiquiátricos; ECR }\end{array}$ \\
\hline $\mathrm{A} 22^{(31)}$ & $\begin{array}{l}\text { China } \\
2019\end{array}$ & Avaliar o efeito do Mindfulness em enfermeiros que atendem AIDS; QE \\
\hline $\mathrm{A} 23^{(32)}$ & Coreia do Sul & $\begin{array}{l}\text { Avaliar a eficácia de um aplicativo para manejo do estresse em } \\
\text { enfermeiros de hospitais universitários; ECR }\end{array}$ \\
\hline
\end{tabular}




\begin{tabular}{|c|c|c|}
\hline & 2019 & \\
\hline $\mathrm{A} 24^{(33)}$ & $\begin{array}{l}\text { USA } \\
2019\end{array}$ & $\begin{array}{l}\text { Avaliar o efeito do } M I N D B O D Y S T R O N G \text { no estresse, ansiedade, sintomas } \\
\text { depressivos, promoção de estilos de vida saudáveis e satisfação no } \\
\text { trabalho em residentes da enfermagem; ECR }\end{array}$ \\
\hline $\mathrm{A} 25^{(34)}$ & Irã & $\begin{array}{l}\text { Avaliar a eficácia do treinamento em regulação emocional na depressão, } \\
\text { ansiedade e estresse de enfermeiros intensivistas; ECR }\end{array}$ \\
\hline $\mathrm{A} 26^{(35)}$ & $\begin{array}{l}\text { USA } \\
2020\end{array}$ & $\begin{array}{l}\text { Avaliar a eficácia de aplicativo de meditação na redução do estresse, } \\
\text { depressão, ansiedade e burnout entre enfermeiras emergencistas; ECR }\end{array}$ \\
\hline $\mathrm{A} 27^{(36)}$ & $\begin{array}{l}\text { USA } \\
2020\end{array}$ & $\begin{array}{l}\text { Avaliar o efeito do treinamento de mindfulness no estresse, ansiedade, } \\
\text { depressão e satisfação no trabalho em enfermeiras hospitalares; ECR }\end{array}$ \\
\hline
\end{tabular}

ECR: Ensaio Clínico Randomizado; QE: Quase experimental.

Fonte: os autores.

O Quadro 3 descreve as intervenções testadas, os instrumentos utilizados para avaliação das variáveis de interesse desta RI e a síntese dos resultados sumarizados.

Quadro 3 - Descrição das intervenções utilizadas, instrumentos de aferição dos desfechos de interesse e sumarização dos resultados. Porto Alegre, RS, Brasil, 2021.

\begin{tabular}{|l|l|l|}
\hline \multicolumn{2}{|l|}{ Desfecho: Estresse } \\
\hline Cod. & Intervenção (amostra) & Instrumentos; Resultados \\
\hline A1 ${ }^{(10)}$ & $\begin{array}{l}\text { GI: programa virtual para manejo do } \\
\text { estresse (52) } \\
\text { GC: sem atividade (52) }\end{array}$ & $\begin{array}{l}\text { Nursing Stress Scale (NSS). Redução do estresse no } \\
\text { GI e GC inalterado (GI: } \bar{x} 2243 \text { vs } 2072 ; p=0.001)\end{array}$ \\
\hline A2 ${ }^{(11)}$ & $\begin{array}{l}\text { GI: Massagem com óleos de lavanda e e } \\
\text { gernio (19) } \\
\text { GC: sem atividade (19) }\end{array}$ & $\begin{array}{l}\text { Escala de Estresse no Trabalho (EET) e Lista de } \\
\text { Sintomas de Estresse (LSS), inalterado no GI e GC } \\
(\text { GI: EET } \bar{x} 5,42 \text { vs 7,74; p=0,9088 e LSS } \bar{x} 52,15 \text { vs } \\
46,31 ; p=0,9202 .)\end{array}$ \\
\hline
\end{tabular}




\begin{tabular}{|c|c|c|}
\hline $\mathrm{A} 3^{(12)}$ & $\begin{array}{l}\text { GI: Mantra associado ao ciclo } \\
\text { menstrual (15) } \\
\text { GC: sem atividade (15) }\end{array}$ & $\begin{array}{l}\text { Variabilidade da Frequência Cardíaca (VFC) e } \\
\text { Cortisol, com aumento da coerência no sistema } \\
\text { nervoso autônomo e redução do cortisol no GI, GC } \\
\text { inalterado (GI: VFC: LF/HF } \bar{x} 1,99 \text { vs } 0,73 ; \mathrm{p}=0,03 \text { e } \\
\text { Cortisol } \bar{x} 268,33 \text { vs } 180,6 ; \mathrm{p}=0,012)\end{array}$ \\
\hline $\mathrm{A} 4^{(13)}$ & $\begin{array}{l}\text { GI: } \quad \text { Terapia } \\
\text { Comportamental (60) } \\
\text { GC: sem atividade (60) }\end{array}$ & $\begin{array}{l}\text { Osipow Questionnaire, com redução do estresse no GI, } \\
\text { GC inalterado ( GI: } \bar{x} 193,58 \text { vs } 145,86 ; \mathrm{p}<0,001 \text { ) }\end{array}$ \\
\hline $\mathrm{A} 5^{(14)}$ & $\begin{array}{l}\text { Protocolo de relaxamento muscular } \\
(16)\end{array}$ & $\begin{array}{l}\text { EET, redução não significativa do estresse }(\bar{x} 60 \text { vs } 55 ; \\
p=0,166)\end{array}$ \\
\hline$A 6^{(15)}$ & $\begin{array}{l}\text { GI: aromaterapia patchouli (25) } \\
\text { GC: aromaterapia óleo amêndoa (25) }\end{array}$ & $\begin{array}{l}\text { Escala análogo visual, redução do estresse }(\bar{x} 7,23 \text { vs } \\
6,03 ; \mathrm{p}<0,001)\end{array}$ \\
\hline $\mathrm{A} 7^{(16)}$ & $\begin{array}{l}\text { GI: Jin Shin Jyutsu Self-Help (18) } \\
\text { GC: sem atividade (23) }\end{array}$ & $\begin{array}{l}\text { Personal and Organizational Quality Assessment, } \\
\text { redução no estresse no GI, GC inalterado } \\
\text { (Estresse organizacional: GI } \bar{x} 3,84 \text { vs } 3,81 ; \mathrm{p}=0,001 \text {. } \\
\text { Estresse emocional: GI } \bar{x}^{2}, 03 \text { vs } 1,57 ; \mathrm{p}<0,00 \text {. } \\
\text { Eestresse físico: GI } \frac{\bar{x}}{3}, 05 \text { vs } 2,35 ; \mathrm{p}=0,001 \text { ) }\end{array}$ \\
\hline $\mathrm{A} 8^{(17)}$ & $\begin{array}{l}\text { GI: Mantra Mahamantra (15) } \\
\text { GC: sem atividade (15) }\end{array}$ & $\begin{array}{l}\text { Cortisol e amilase salivar, redução nos níveis } \\
\text { hormonais no GI, GC inalterado (GI: cortisol } \bar{x} 268,33 \\
\text { vs } 180,6 ; \mathrm{p}=0.01 \text {. Amilase } \bar{x} 150,13 \text { vs } 117,2 ; \mathrm{p}= \\
0.04)\end{array}$ \\
\hline A9 ${ }^{(18)}$ & $\begin{array}{l}\text { GI: programa educativo } \\
\text { gerenciamento do estresse (40) } \\
\text { GC: } \text { sem atividade (40) }\end{array}$ & $\begin{array}{l}\text { Job Stress Questionnaire, redução do estresse no GI, } \\
\text { GC inalterado (GI: } \bar{x}^{3}, 64 \text { vs } 3,10 ; p=0,03 \text { ) }\end{array}$ \\
\hline $\mathrm{A}^{10^{(19)}}$ & $\begin{array}{l}\text { GI: treinamento educativo sobre } \\
\text { gerenciamento do estresse (40) }\end{array}$ & $\begin{array}{l}\text { Osipow Questionnaire, sem efeito no GI e no GC (GI: } \\
\bar{x} 182,52 \text { vs } 186,22 ; \mathrm{p}=0,48)\end{array}$ \\
\hline
\end{tabular}




\section{\begin{tabular}{|l|l}
\hline GC: sem atividade (40) \\
\hline
\end{tabular}}

\section{2) Desfecho: Ansiedade}

\begin{tabular}{|c|c|c|}
\hline $\mathrm{A} 11^{(20)}$ & $\begin{array}{l}\text { GI(1): Auriculoterapia agulhas (45) } \\
\text { GI(2): Auriculoterapia sementes (45) } \\
\text { GI(3): Fita adesiva (45) } \\
\text { GC: Sem tratamento (45) }\end{array}$ & $\begin{array}{l}\text { Inventário de Ansiedade Traço-Estado (IDATE), } \\
\text { houve efeito na redução da ansiedade nos três GI, GC } \\
\text { inalterado (GI (1) } d \text { de Cohen de } 1,08, \mathrm{GI}(2) 0,71 \text { e } \\
\text { GI(3) 0,63; p=0,028) }\end{array}$ \\
\hline $\mathrm{A} 12^{(21)}$ & $\begin{array}{l}\text { GI: Aulas de música (24) } \\
\text { GC sem atividade (19) }\end{array}$ & $\begin{array}{l}\text { Hospital Anxiety and Depression Scale (HADS), } \\
\text { redução da ansiedade no GI, GC inalterado }(\bar{x} 17 \times \\
14,5 ; \mathrm{p}=0,04 ; d=0,836)\end{array}$ \\
\hline $\mathrm{A} 13^{(22)}$ & $\begin{array}{l}\text { GI: Mindfulness (40) } \\
\text { GC: panfletos acerca do tema (40) }\end{array}$ & $\begin{array}{l}\text { HADS e Geralized Anxiety Disorder Scale (GADS), } \\
\text { sem efeito GI e GC; } p=0,566 \\
\text { HADS ( } \bar{x} 3,89 \text { vs } 4,06 \text { no GI e } 4,03 \text { vs } 3,91 \text { no GC) } \\
\text { GADS }(\bar{x} 3,05 \text { vs } 4,21 \text { no GI e } 3,16 \text { vs } 3,31 \text { no GC) }\end{array}$ \\
\hline $\mathrm{A} 14^{(23)}$ & Logoterapia (40) & $\begin{array}{l}\text { Death Anxiety Questionnaire, redução da ansiedade } \\
(\bar{x} 51 \text { vs } 45 ; \mathrm{p}=0,003)\end{array}$ \\
\hline $\mathrm{A} 15^{(24)}$ & Colorir mandalas pré-desenhadas (40) & $\begin{array}{l}\text { STAI-Form e VFC, efeito somente na VFC (STAI: } d= \\
0,055 \text { e VFC } d=0,37 \text { ) }\end{array}$ \\
\hline
\end{tabular}

\section{3) Desfecho: Estresse e Ansiedade}

\begin{tabular}{|c|c|c|}
\hline $\mathrm{A} 16^{(25)}$ & Mindfulness e meditação (13) & $\begin{array}{l}\text { IDATE e EET, redução da ansiedade (Trait) e estresse } \\
\text { (IDATE: State } \bar{x} 45,08 \text { vs } 41,38 ; \mathrm{p}=0,254 \text {; Trait } \bar{x} 48,92 \\
\left.\text { vs } 41,38 ; \mathrm{p}=0,049 ; \text { EET } \bar{x}^{23,85} \text { vs } 17,08 ; \mathrm{p}=0,001\right)\end{array}$ \\
\hline $\mathrm{A} 17^{(26)}$ & $\begin{array}{l}\text { GI: Aromaterapia com óleo de } \\
\text { lavanda (43) } \\
\text { GC: óleo de girassol (42) }\end{array}$ & $\begin{array}{l}\text { Perceived Stress Scale (PSS) e IDATE, sem efeito nos } \\
\text { desfechos. (GI: } \bar{x} 27,5 \text { vs } 27,3 ; \mathrm{p}=0,530) \text {; State (GI: } \\
\bar{x} 31,6 \text { vs } 31,9 ; \mathrm{p}=0,348)\end{array}$ \\
\hline $\mathrm{A} 18^{(27)}$ & Curso sobre estresse e resiliência e & PSS e GADS, redução nos desfechos, $29,8 \%$ no \\
\hline
\end{tabular}




\begin{tabular}{|c|c|c|}
\hline & leitura de um livro (50) & estresse $(\mathrm{p}=0,001)$ e $45,2 \%$ na ansiedade $(\mathrm{p}<0,001)$ \\
\hline $\mathrm{A} 19^{(28)}$ & $\begin{array}{l}\text { GI: Relaxamento e exercícios de } \\
\text { respiração (13) } \\
\text { GC: sem atividade (13) }\end{array}$ & $\begin{array}{l}\text { IDATE e Semantic differential Scales, sem efeito nos } \\
\text { desfechos (GI: State } p=0,71 \text {; Trait } \mathrm{p}=0,40 \text {. Stress } \\
\mathrm{p}=0,10)\end{array}$ \\
\hline $\mathrm{A} 20^{(29)}$ & $\begin{array}{l}\text { GI: Mindfulness e workshop } \\
\text { educacional de manejo do estresse e } \\
\text { ansiedade (46) } \\
\text { GC: } \text { sem atividade(45) }\end{array}$ & $\begin{array}{l}\text { DASS, sem efeito nos desfechos (GI: ansiedade } \\
p=0,165 \text {; estresse } p=0,136)\end{array}$ \\
\hline $\mathrm{A} 21^{(30)}$ & $\begin{array}{l}\text { GI: Mindfulness (50); GC: sem } \\
\text { atividade (50) }\end{array}$ & $\begin{array}{l}\text { The self-rating anxiety scale (SAS) e NSS, redução } \\
\text { dos desfechos no GI, GC inalterado (SAS GI: } \bar{x}^{44,8} \\
\text { vs } 36,4 ; \mathrm{p}<0,001 \text { e NSS GI: } \bar{x} 83,9 \text { vs } 68,2 ; \mathrm{p}<0,001 \text { ) }\end{array}$ \\
\hline $\mathrm{A} 22^{(31)}$ & Mindfuln & 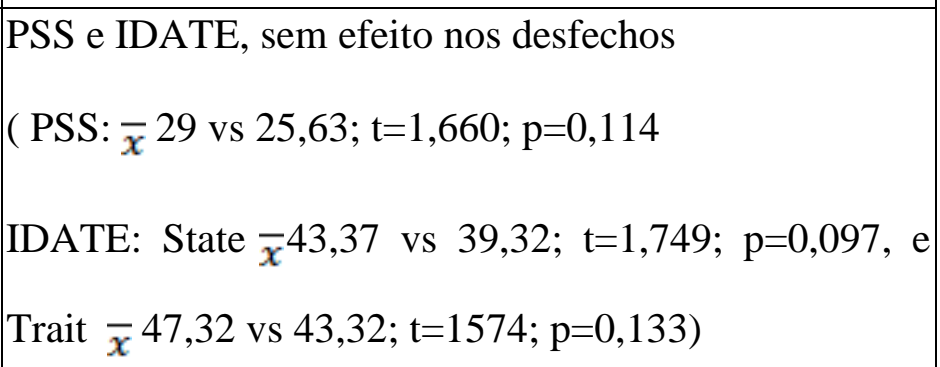 \\
\hline $\mathrm{A} 23^{(32)}$ & $\begin{array}{l}\text { GI: música focada na cura, meditação, } \\
\text { métodos de respiração e yoga e } \\
\text { orientações sobre saúde mental (26) } \\
\text { GC: sem atividade (30) }\end{array}$ & $\begin{array}{l}\text { PSS e GAD, redução do estresse no GI, ansiedade sem } \\
\text { redução significativa } \\
\text { PSS GI: } \bar{x}^{20} \text { vs } 18,50 ; p=0,035 \\
\left.\text { GAD GI: } \bar{x}^{4,26} \text { vs } 4,23 ; \mathrm{p}=0,966\right)\end{array}$ \\
\hline $\mathrm{A} 24^{(33)}$ & Programa Mindbodystrong (89) & $\begin{array}{l}\text { PSS e GADS, redução dos desfechos (PSS: } F=5,459 ; \\
\mathrm{p}=0,022 \text { e GADS: }(F=10,40 ; \mathrm{p}=0,002)\end{array}$ \\
\hline $\mathrm{A} 25^{(34)}$ & $\begin{array}{l}\text { GI: treinamento para regulação } \\
\text { emocional (30) } \\
\text { GC: sem atividade (30) }\end{array}$ & $\begin{array}{l}\text { DASS, redução dos desfechos no GI, GC inalterado ( } \\
\text { GI Ansiedade } F=4,10 ; p=0,04 \text { e Estresse } F= \\
5,33, p=0,03)\end{array}$ \\
\hline $\mathrm{A} 26^{(35)}$ & $\begin{array}{l}\text { GI: Aplicativo de meditação "Stress } \\
\text { Free Now Meditations for Healers" }\end{array}$ & $\begin{array}{l}\text { PSS e Beck Anxiety Inventory (BAI), redução no } \\
\text { estresse e ansiedade no GI, GC inalterado (PSS GI p= }\end{array}$ \\
\hline
\end{tabular}




\begin{tabular}{|l|l|l|}
\hline & $\begin{array}{l}\text { (13) } \\
\text { GC: } \text { sem intervenção (12) }\end{array}$ & $0,032 ;$ GC $\mathrm{p}=0,635$ e BAI GI $\mathrm{p}=0,014 ; \mathrm{GC} \mathrm{p}=0,425)$ \\
\hline A27(36) & $\begin{array}{l}\text { GI: Mindfulness (118) } \\
\text { GC: sem atividade (106) }\end{array}$ & $\begin{array}{l}\text { DASS, redução da ansiedade, sem efeito no estresse } \\
\text { quando comparado GI vs GC (Ansiedade } \mathrm{p}=0,037 \text { e } \\
\text { estresse } \mathrm{p}=0,159)\end{array}$ \\
\hline
\end{tabular}

GI: Grupo intervenção; GC: Grupo controle.

Fonte: os autores.

Verificou-se intervenções com resultado estatisticamente significativo na redução dos desfechos em $15(55,5 \%)$ das publicações. Autores de 16 (59,3\%) artigos referiram que a amostra reduzida foi a principal limitação do estudo.

\section{DISCUSSÃO}

Identificou-se a utilização de uma grande variedade de intervenções, com atividades tradicionais

como auriculoterapia $^{(20)}$ e $\operatorname{aromaterapia}^{(15,26)}$, algumas com cunho cultural, como mantras ${ }^{(12,17)}$ e outras inseridas em programas e protocolos, como o Mindbodystrong ${ }^{(33)}$, o Relation Response ${ }^{(28)}$ e o Jin Shin Jyutsu ${ }^{(16)}$. Emergiram atividades elaboradas, programas de redução do estresse e ansiedade que já se encontram comercializados ou com marca registrada, assim como atividades simples e de baixo custo. A heterogeneidade nas intervenções inviabilizou a análise comparativa entre as publicações.
O Mindfulness foi a terapia mais utilizada $^{(22,29-31,36)}$, com resultado positivo em três pesquisas. Estudo que analisou intervenções utilizadas para promover o bemestar no trabalho verificou que intervenções baseadas em mindfulness e meditação são as mais eficazes para melhorar a saúde e o desempenho ocupacional ${ }^{(37)}$.

Houve ausência de resultado satisfatório em três estudos para redução do estresse $^{(11,14,19)}$, um para ansiedade ${ }^{(22)}$, cinco para estresse e ansiedade ${ }^{(26,28,29,31,36)}$, além de dois resultados parcialmente satisfatórios $(24,32)$. Os autores citam como limitação o fato de terem trabalhado com amostras pequenas. No entanto, esta RI identificou pesquisas ${ }^{(21,35)}$ com amostras com menos de 30 indivíduos e com resultado estatisticamente satisfatório, sugerindo que distintas variáveis possam ter interferido no resultado da intervenção ou que o instrumento utilizado não foi sensível para captar as modificações nos trabalhadores.

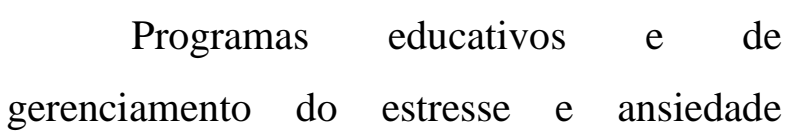


apareceram em cinco estudos $^{(10,18,19,27,34)}$, com resultado positivo em quatro deles. Entendese que o estresse relacionado à profissão pode ser atenuado, mas não extinto. Por esse motivo, programas educacionais de gerenciamento do estresse são vistos como benéficos e necessários, reduzindo efeitos adversos do trabalho ${ }^{(18)}$. Estudo ${ }^{(38)}$ identificou em uma avaliação realizada com trabalhadores de diversos ramos que $\mathbf{o}$ relaxamento e a meditação foram as terapias mais utilizadas, entretanto, os profissionais que utilizaram abordagem cognitivas e educacionais obtiveram melhores resultados.

Apenas três pesquisas ${ }^{(12,17,24)}$ utilizaram marcadores biológicos para avaliar o efeito da intervenção. A avaliação de marcadores biológicos oferece um panorama fisiológico das condições do indivíduo quando ocorre exposição a eventos estressantes, sendo considerada como padrão ouro. A variabilidade da frequência cardíaca, é um marcador biológico não invasivo que fornece resultados em tempo real, com a utilização de softwares disponíveis no mercado $^{(12,24) .}$ Por este motivo, poderiam ser mais utilizadas em pesquisas com estresse e ansiedade.

Esta RI direciona para a necessidade da realização de pesquisas robustas na área, a partir da identificação das intervenções utilizadas e dos resultados obtidos em profissionais de enfermagem, podendo servir como subsídio para encorajar as instituições a organizarem programas contínuos e estruturados de gerenciamento do estresse e ansiedade. Ressalta-se que as publicações selecionadas sugerem a individualização do tratamento, apostando no reconhecimento das singularidades de cada trabalhador ou nas características de um grupo, utilizando as distintas possibilidades existentes.

\section{CONCLUSÃO}

Esta revisão identificou intervenções utilizadas para redução do estresse ocupacional e ansiedade em profissionais da enfermagem, contribuindo para o conhecimento e promoção da saúde ocupacional. Entretanto, verificou-se que a mesma intervenção obteve resultados divergentes em populações distintas, sugerindo uma análise detalhada dos grupos, teste piloto, e individualização da intervenção conforme a necessidade. Salienta-se a importância do investimento em programas com múltiplas atividades, que incentivem o autoconhecimento.

\section{REFERÊNCIAS}

1. Moraes Filho IM, José de Almeida R. Estresse ocupacional no trabalho em enfermagem no Brasil: uma revisão integrativa. Rev Bras em promoção da Saúde[Internet]. 2016 [acesso em 15 out 2020];29(3). Disponível em: https://periodicos.unifor.br/RBPS/article/view 


\section{$/ 4645$}

2. Bezerra MAA, Julião JF, Bezerra GG de O, Lopes CR, Bottcher LB. Ansiedade, estresse e níveis de atividade física em escolares. Rev Interdiscip Encontro das Ciências - RIEC [Internet]. 2019 [acesso em 14 jul 2020];2(1). Disponível em http://fvs.edu.br/riec/index.php/riec/article/vie w/56/42

3. Guimarães AMV, Neto ACS, Vilar ATS, Almeida BG da C, Fermoseli AFO, Albuquerque CMF. Transtornos De Ansiedade: Um Estudo De Prevalência Sobre As Fobias Específicas e a Importância da Ajuda Psicológica. Cad Grad - Ciências Biológicas e da Saúde [Internet]. 2015 [acesso em 15 out 2020];3(1). Disponível em: https://periodicos.set.edu.br/fitsbiosaude/articl e/view/2611

4. Associação Nacional de Medicina do Trabalho. Estresse e burnout: por que a saúde emocional no trabalho importa? [Internet]. 2019 [acesso em 1 fev 2021]. Disponível em: https://www.anamt.org.br/portal/2019/07/12/e stresse-e-burnout-por-que-a-saude-emocionalno-trabalho-importa/

5. Uchôa L, Veloso P, Marcilon L, Laurindo B, Sousa LRP, Veloso C. Prevalence of anxiety in nursing professionals of urgency and emergency. Rev Enferm UFPE Line [Internet]. 2016 [acesso em 12 out 2020];10(11). Disponível em: http://doi.org/10.5205/1981-8963v10i11a11479p3969-3976-2016

6. Freitas M A, Silva Junior CO, Machado AD, Valente COT. Plano de Ação para Redução do Estresse em Enfermeiros de um Hospital Universitário. Enferm Atual [Internet]. 2018 [acesso em 19 jul 2020];1(8). Disponível em https://revistaenfermagematual.com.br/index. php/revista/article/view/268/160

7. Paula CC, Padoin SM de M, Galvão CM.
Revisão integrativa como ferramenta para tomada de decisão na prática em saúde. Vol. I. Porto Alegre: Moriá Editora; 2018. 52-76 p.

8. Santos CMDC, Pimenta CADM, Nobre MRC. The PICO strategy for the research question construction and evidence search. Rev Lat Am Enfermagem [Internet]. 2007 [acesso em 25 jul 2020];15(3). Disponível em https://doi.org/10.1590/S010411692007000300023

9. Moher D, Liberati A, Tetzlaff J, Altman DG, Altman D, Antes G. Preferred reporting items for systematic reviews and metaanalyses: The PRISMA statement. PLoS Medicine. Public Library of Science [Internet]. 2009 [acesso em 6 dez 2020]. 6; p. e1000097. Disponível em : https://doi.org/10.1371/journal.pmed.1000097

10. Hersch RK, Cook RF, Deitz DK, Kaplan S, Hughes D, Friesen MA. Reducing nurses' stress: A randomized controlled trial of a web-based stress management program for nurses. Appl Nurs Res [Internet]. 2016 [acesso 1 mai 2020];32. Disponível em: https://doi.org/10.1016/j.apnr.2016.04.003

11. Montibeler J, Domingos TS, Braga EM, Gnatta JR, Kurebayashi LFS, Kurebayashi AK. Effectiveness of aromatherapy massage on the stress of the surgical center nursing team: A pilot study. Rev da Esc Enferm [Internet]. 2018 [acesso em 2 ago 2020];52. Disponível em https://dx.doi.org/10.1590/S1980220X2017038303348

12. Sekar L, Niva W, Maheshkumar K, Thangavel G, Manikandan A, Silambanan S. Effect of Mahamantra Chanting on Autonomic and Cognitive Functions-An Interventional Study. J Clin DIAGNOSTIC Res [Internet]. 2019 [acesso em 27 abr 2020]; Disponível em: https://doi.org/10.7860/JCDR/2019/41236.12 877

13. Fadaei M, Torkaman M, Heydari N, Kamali M, Ghodsbin F. Cognitive behavioral therapy for occupational stress among the 
intensive care unit nurses. Indian J Occup Environ Med [Internet]. 2020 [acesso em 27 fev 2021];24(3). Disponível em: doi:10.4103/ijoem.IJOEM_286_19

14. Silveira EA, Batista KM, Grazziano ES, Bringuete ME de O, Lima E de FA. Efeito do relaxamento muscular progressivo no estresse e bem-estar no trabalho de enfermeiros hospitalar. Enfermería Glob [Internet]. 2020 [acesso em 27 fev 2021];19(2). Disponível em: https://doi.org/10.6018/eglobal.396621

15. Shin YK, Lee SY, Lee JM, Kang P, Seol GH. Effects of short-term inhalation of patchouli oil on professional quality of life and stress levels in emergency nurses: A randomized controlled trial. J Altern Complement Med [Internet]. 2020 [acesso em 27 fev 2021];26(11). Disponível em: https://doi.org/10.1089/acm.2020.0206

16. Millspaugh J, Errico C, Mortimer S, Kowalski MO, Chiu S, Reifsnyder C. Jin Shin Jyutsu ${ }^{\circledR}$ Self-Help Reduces Nurse Stress: A Randomized Controlled Study. J Holist Nurs [Internet]. 2020 [acesso em 21 fev 2021]; Disponível em: https://doi.org/10.1177/0898010120938922

17. Niva WJ, Sekar L, Manikandan A, K MK, T G, Shriraam V, et al. Mahamantra chanting as an effective intervention for stress reduction among nursing professionals:A randomized controlled study. Adv Integr Med[Internet]. 2020 [acesso em $21 \mathrm{fev}$ 2021];8(1). Disponível em: https://doi.org/10.1016/j.aimed.2020.05.007

18. Valipour S, Aazami S, Mozafari M. The Effect of Training Intervention on the Level of Stress Management Skills in Novice Nurses Working at Educational Hospitals in Ilam Province. J Cardiovasc Dis Res [Internet]. 2020[acesso em $21 \mathrm{fev}$ 2021];11(4). Disponível em: doi: 10.31838/jcdr.2020.11.04.17

19. Ravari AK, Farokhzadian J, Nematollahi M, Miri S, Foroughameri G. The Effectiveness of a Time Management Workshop on Job Stress of Nurses Working in Emergency Departments: An Experimental
Study. J Emerg Nurs [Internet]. 2020 [acesso em 27 fev 2021 ];46(4). Disponível em: https://doi.org/10.1016/j.jen.2020.03.013

20. Kurebayashi LFS, Turrini RNT, De Souza TPB, Marques CF, Rodrigues RTF, Charlesworth K. Auriculoterapia para redução de ansiedade e dor em profissionais de enfermagem: Ensaio clínico randomizado. Rev Lat Am Enfermagem [Internet]. 2017 [acesso em 2 ago 2020];25(0). Disponível em: http://dx.doi.org/10.1590/15188345.1761.2843

21. Ploukou S, Panagopoulou E. Playing music improves well-being of oncology nurses. Appl Nurs Res[Internet]. 2018 [acesso em 21 fev 2021]1(39). Disponível em: https://doi.org/10.1016/j.apnr.2017.11.007

22. Watanabe N, Horikoshi M, Shinmei I, Oe Y, Narisawa T, Kumachi $M$, et al. Brief mindfulness-based stress management program for a better mental state in working populations - Happy Nurse Project: A randomized controlled trial. J Affect Disord [Internet]. 2019 May 15 [cited 2020 Aug 3];251:186-94. Disponível em: https://doi.org/10.1186/s13063-015-0554-Z

23. Sheykhi M, Naderifar M, Firouzkohi M, Abdollahimohammad A. Effect of group logotherapy on death anxiety and occupational burnout of special wards nurses. Med Sci [Internet]. 2019 [acesso em 27 abr 2020];23(98). Disponível em: www.discoveryjournals.org

24. Maguire P, Coughlan A, Drayton D, Lacko H, Reich J, Hatfield L. The effect of coloring mandalas on the anxiety of medicalsurgical nurses and nursing support staff. Medsurg Nurs [Internet]. 2020 [acesso em 27 abr 2020];29(3)Disponível em: https://oce.ovid.com/article/00008484202005000-00010/HTML

25. Santos TM, Kozasa EH, Carmagnani IS, Tanaka LH, Lacerda SS, Nogueira-Martins LA. Positive Effects of a Stress Reduction Program Based on Mindfulness Meditation in Brazilian Nursing Professionals: Qualitative and Quantitative Evaluation. EXPLORE 
[Internet]. 2016 [acesso em 27 abr 2020];12(2). Disponível em:https://linkinghub.elsevier.com/retrieve/pi i/S1550830715002232

26. Baris Eren N, Oztunc G. The Effects of Aromatherapy on the Stress and Anxiety Levels of Nurses Working in Intensive Care Units. International Journal of Caring[Internet]. 2018 [acesso em 1 mai 2020];10(3). Disponível em: www.internationaljournalofcaringsciences.org

27. Magtibay DL, Chesak SS, Coughlin K, Sood A. Decreasing Stress and Burnout in Nurses: Efficacy of Blended Learning With Stress Management and Resilience Training Program. J Nurs Adm [Internet]. 2017 [acesso em 2 ago 2020];47(7-8). Disponível em: doi: 10.1097/NNA.0000000000000501

28. Calisi CC. The Effects of the Relaxation Response on Nurses' Level of Anxiety, Depression, Well-Being, Work-Related Stress, and Confidence to Teach Patients. J Holist Nurs [Internet]. 2017 [acesso em 1 mai 2020];35(4). Disponível em: https://doi.org/10.1177/0898010117719207

29. Slatyer S, Craigie M, Heritage B, Davis S, Rees C. Evaluating the Effectiveness of a Brief Mindful Self-Care and Resiliency (MSCR) Intervention for Nurses: a Controlled Trial. Mindfulness[Internet]. 2018 [acesso em 1 mai 2020]9(2). Disponível em: https://link.springer.com/article/10.1007/s126 71-017-0795-x

30. Yang J, Tang S, Zhou W. Effect of mindfulness-based stress reduction therapy on work stress and mental health of psychiatric nurses. Psychiatr Danub[Internet]. 2018 [acesso em 1 mai 2020]; 30(2). Disponível em: doi: 10.24869/psyd.2018.189.

31. Pan C, Wang H, Chen M, Cai Y, Xiao C, Tang Q, et al. Mindfulness-based intervention for nurses in AIDS care in China: A pilot study. Neuropsychiatr Dis Treat [Internet]. 2019 [acesso em 1 mai 2020];15. Disponível em: doi: 10.2147/NDT.S223036

32. Hwang WJ, Jo HH. Evaluation of the effectiveness of mobile app-based stressmanagement program: A randomized controlled trial. Int $\mathbf{J}$ Environ Res Public Health [Internet]. 2019 [acesso em 1 mai 2020]];16(21). Disponível https://doi.org/10.3390/ijerph16214270

33. Sampson M, Melnyk BM, Hoying J. Intervention Effects of the MINDBODYSTRONG Cognitive Behavioral Skills Building Program on Newly Licensed Registered Nurses' Mental Health, Healthy Lifestyle Behaviors, and Job Satisfaction. J Nurs Adm [Internet]. 2019 [acesso em 3 ago 2020 ];49(10). Disponível em: doi: 10.1097/NNA.0000000000000792

34. Kharatzadeh H, Alavi M, Mohammadi A, Visentin D, Cleary M. Emotional regulation training for intensive and critical care nurses. Nurs Heal Sci [Internet]. 2020 [acesso em 2 ago cited 2020];22(2). Disponível em: https://onlinelibrary.wiley.com/doi/abs/10.111 1/nhs. 12679

35. Lambert KG, Aufricht WR, Mudie D, Brown LH. Does a phone-based meditation application improve mental wellness in emergency medicine personnel? [Internet]. Vol. 38, American Journal of Emergency Medicine. W.B. Saunders[Internet]; 2020 [cited 2021 Feb 27]. p. 2740-1. Disponível em: doi: 10.1016/j.ajem.2020.04.058

36. Ghawadra SF, Lim Abdullah K, Choo WY, Danaee M, Phang CK. The effect of mindfulness-based training on stress, anxiety, depression and job satisfaction among ward nurses: A randomized control trial. J Nurs Manag [Internet]. 2020 [acesso 28 fev 2021 ];28(5). Disponível em: https://onlinelibrary.wiley.com/doi/abs/10.111 1/jonm.13049

37. Ravalier JM, Wegrzynek P, Lawton S. Systematic review: Complementary therapies and employee well-being. Occup Med [Internet]. 2016 [acesso 13 jun 2020];66(6). Disponível em: https://doi.org/10.1093/occmed/kqw047

38. Simonelli L. Estresse ocupacional e alternativas de intervenção: um estudo 
bibliométrico. Res Soc Dev [Internet]. 2020 [acesso em 3 fev 2021;9(3). Disponível em: https://rsdjournal.org/index.php/rsd/article/vie w/2401

Submissão: 2021-06-13

Aprovado: 2021-07-28

Autor correspondente: Andréia Barcellos Teixeira Macedo Rua São Francisco, 528, Bairro Nossa Senhora das Graças, Canoas, RS CEP 92025-410 Fone (51) 993129487 abtmacedo@gmail.com 